\title{
The Final Stage of Cholinergic Differentiation Occurs Below Inner Hair Cells During Development of the Rodent Cochlea
}

\author{
Adam L. Bergeron, ${ }^{1,2}$ Angela Schrader, ${ }^{3}$ Dan Yang, ${ }^{1}$ Abdullah A. Osman, ${ }^{1}$ \\ AND Dwayne D. Simmons ${ }^{1,2,3}$ \\ ${ }^{1}$ Department of Otolaryngology, Washington University School of Medicine, Box 8115660 South Euclid Ave., St. Louis, \\ MO 63110, USA \\ ${ }^{2}$ Program in Neuroscience, Washington University School of Medicine, St. Louis, MO 63110, USA \\ ${ }^{3}$ Department of Anatomy and Neurobiology, Washington University School of Medicine, St. Louis, MO 63110, USA
}

Received: 7 June 2005; Accepted: 31 August 2005; Online publication: 15 October 2005

\begin{abstract}
To gain further insights into the cholinergic differentiation of presynaptic efferent terminals in the inner ear, we investigated the expression of the highaffinity choline transporter (ChT1) in comparison to other presynaptic and cholinergic markers. In the adult mammalian cochlea, cholinergic axons from medial olivocochlear (OC) neurons form axosomatic synapses with outer hair cells (OHCs), whereas axons from lateral OC neurons form axodendritic synapses on afferent fibers below inner hair cells (IHCs). Mouse brain and cochlea homogenates reveal at least two ChT1 isoforms: a nonglycosylated $\sim 73 \mathrm{kDa}$ protein and a glycosylated $\sim 45 \mathrm{kDa}$ protein. In mouse brain, ChT1 is preferentially expressed by neurons in periolivary regions of the superior olive consistent with the location of medial OC neurons. In the adult mouse cochlea, ChT1-positive terminals are located almost exclusively below OHCs consistent with a medial OC innervation. Between postnatal day 2 (P2) and P4, ChT1-positive terminals are below IHCs and occur after the expression of growthassociated protein 43, synapsin, and the vesicular acetylcholine transporter. By P15, ChT1-positive terminals are mostly on OHCs. Accounting for dif-
\end{abstract}

Correspondence to: Dwayne D. Simmons - Department of Otolaryngology • Washington University School of Medicine • Box 8115660 South Euclid Ave., St. Louis, MO 63110, USA. Telephone: (314) 747-7272; fax: (314) 747-7230; email: dsimmons@wustl.edu ferences in gestational age, the developmental expression of ChT1 in the rat cochlea is similar to the mouse. However, in older rats ChT1-positive terminals are below IHCs and OHCs. In both rat and mouse, our observations indicate that the onset of ChT1 expression occurs after efferent terminals are below IHCs and express other presynaptic and cholinergic markers. In the mouse, but not in the rat, ChT1 may preferentially identify medial OC neurons.

Keywords: olivocochlear efferent neurons, auditory, inner ear, mouse, rat

\section{INTRODUCTION}

Acetylcholine is a primary neurotransmitter for synaptic transmission between efferent olivocochlear (OC) axons and cochlear hair cells within the mammalian inner ear. Efferent OC neurons originate in the brainstem superior olivary complex where they can be divided into at least two populations based on location, morphology, and projections to the cochlea. In most rodents, lateral OC neurons have somata located within the lateral superior olive and axons that terminate on afferent nerve fibers innervating inner hair cells (IHCs). In contrast, medial OC neurons reside mostly in ventral periolivary regions and have axons that terminate directly on outer hair cells (OHCs) (Warr and Guinan 1979; Simmons 2002; Maison et al. 2003). In studies of the superior 
olivary complex, both medial and lateral efferent OC neurons express the biosynthetic enzymes required for acetylcholine synthesis including choline acetyltransferase (ChAT), vesicular acetylcholine transporter (VAChT), and acetylcholinesterase (AChE) (Yao and Godfrey 1998; Simmons et al. 1999; Simmons 2002). Calcitonin gene-related peptide (CGRP) is exclusively expressed by lateral efferents in some species such as the hamster (Simmons and Raji-Kubba 1993; Simmons et al. 1997; Raji-Kubba et al. 2002), but in other species such as the mouse, CGRP appears to be expressed by both lateral and medial efferents (Maison et al. 2003). To date, an exclusive marker for medial efferent OC neurons has not been identified.

De novo synthesis of acetylcholine from acetyl-CoA and choline is required for rapid synaptic transmission between neurons and their targets at central and peripheral synapses. Consequently, choline transport within presynaptic axon terminals is required to maintain a sufficient pool of substrate for neurotransmitter synthesis and choline uptake is thought to be the rate-limiting step for acetylcholine synthesis (Haga and Noda 1973; Kuhar and Murrin 1978; Tucek 1985). In cochlear studies, a hemicholinium-3 sensitive release of acetylcholine has been known for some time (Galley et al. 1973; Comis and Guth 1974; Comis et al. 1974). Recently, the cDNA for a hemicholinium-3-sensitive, high-affinity choline transporter (ChT1) was characterized in C. elegans (Okuda et al. 2000), mouse (Apparsundaram et al. 2001), rat (Okuda et al. 2000), and man (Apparsundaram et al. 2000; Okuda and Haga 2000). The expression of ChT1 in presynaptic motor neurons colocalizes with postsynaptic nicotinic acetylcholine receptors on muscle and is found predominately associated with presynaptic vesicles (Lips et al. 2002; Ferguson et al. 2003; Okuda and Haga 2003; Nakata et al. 2004).

In this study, we compared the developmental expression and localization of ChT1 in the mouse brainstem and cochlea to other cholinergic (e.g., vesicular acetylcholine transporter) and presynaptic terminal markers. Growth-associated protein 43 (GAP43) was compared to ChT1 in embryonic and early postnatal animals, and SNARE complex proteins, synapsin and SNAP25, were compared to ChT1 in older postnatal and adult animals. To test the generality of findings in the mouse across species, we also compared the development of ChT1 immunoreactivity in the mouse cochlea to the rat cochlea. Our observations suggest that high-affinity choline transport is involved in the synthesis of acetylcholine within the efferent OC system and is expressed after efferent axons arrive in the organ of Corti. At least in the mouse, our data also indicate that ChT1 may preferentially label a subset of OC neurons.

\section{MATERIALS AND METHODS}

\author{
Experimental subjects
}

ICR and C57BL mice [ages: embryonic day 18 (E18), postnatal day 0 (P0), P2, P4, P6, P10, P15, P20, P50, P180] and Sprague-Dawley rats [ages: E18, P0, P2, P4, P6, P10, P21] were either bred in-house or obtained from Harlan Laboratories (Indianapolis, IN, USA). Appearance of the vaginal plug was representative of gestation day 1 (E1). For embryonic and postnatal animals, the crown-to-rump lengths were measured and used to verify the developmental stage of each animal. The Washington University Institutional Animal Care and Use Committee approved all experimental procedures.

\section{Tissue preparation}

Adult and postnatal animals were euthanized with an injection (i.p.) of Nembutal $(100 \mathrm{mg} / \mathrm{kg}$; Abbott Laboratories, Chicago, IL, USA). Following anesthesia, embryos and neonatal animals up to P2 were fixed by immersion in either $2 \%$ or $4 \%$ paraformaldehyde buffered with $0.1 \mathrm{M}$ sodium phosphate in

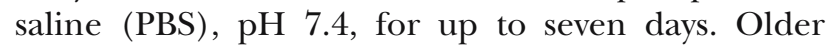
postnatal animals (i.e., P3 and greater) were perfused transcardially with either $2 \%$ or $4 \%$ paraformaldehyde in PBS. Following fixation, brainstems and temporal bones were removed and immediately immersed in fixative. Temporal bones were further dissected to remove the cochlea. In animals P10 and older, cochleae were decalcified in $10 \%$ EDTA (w/v) overnight at $4^{\circ} \mathrm{C}$.

The organ of Corti was prepared in one of two ways. For whole-organ preparations, the organ of Corti was microdissected to remove adhering membranes and the organs transferred to $1.5-\mathrm{mL}$ tubes containing 0.1 M PBS. For frozen section preparation, the cochlea was embedded in OCT (Sakura USA, Torrance, CA, USA) and serial sections cut at 10-12 $\mu \mathrm{m}$ in the modiolar (radial) plane on a cryostat. Sections were transferred to Superfrost Plus slides (VWR Scientific, West Chester, PA, USA) and slides were stored at $-20^{\circ} \mathrm{C}$ prior to processing for immunocytochemistry. Brainstems were completely dissected and sectioned on a Vibratome at $50-\mu \mathrm{m}$ thickness.

\section{Western blot}

To isolate total protein, P6 ICR mouse and P6 rat brains and cochleae were dissected, frozen in liquid N2, and pulverized. Samples were denatured for 15 min at $37^{\circ} \mathrm{C}$ in Laemmli sample buffer [ $1 \%$ sodium dodecyl sulfate (SDS), $31.25 \mathrm{mM}$ Tris, $\mathrm{pH}$ 6.8, 5\% glycerol, $200 \mathrm{mM}$ 2-mercaptoethanol; Laemmli 
1970], resolved by standard SDS-polyacrylamide gel electrophoresis (PAGE) (15\%; $5 \mu \mathrm{g} 5$ protein/lane), and transferred electrophoretically to polyvinylidene difluoride (PVDF) membranes (Amersham Biosciences, Arlington Heights, IL, USA). Protein samples were quantitated using the bicinchoninic acid (BCA) assay (Pierce, Rockford, IL, USA) with bovine serum albumin as a standard.

PVDF membranes were blocked in wash Trisbuffered saline with Tween (TBS-T, 0.1 M TBS, $0.5 \%$ Tween 20) containing $5 \%$ nonfat dry milk for $1 \mathrm{~h}$ and then incubated overnight at $4^{\circ} \mathrm{C}$ with a rabbit polyclonal antiserum to the $\mathrm{C}$ terminus of the highaffinity ChT1 (1:500; a generous gift from H. Misawa, Department of Neurology, Tokyo Metropolitan Institute for Neuroscience, Tokyo, Japan). Blots were incubated with goat anti-rabbit HRP-conjugated secondary antibody (1:50,000; Chemicon, Temecula, CA, USA) and after extensive washing in PBS with Tween (PBS-T, 0.1 M PBS, 0.5\% Tween-20) the signal was detected with SuperSignal West Chemiluminescence Reagent (Pierce).

The predicted size of the ChT1 protein was calculated by entering the mouse amino acid sequence for ChT1 (accession number Q8BGY9) into a protein molecular weight calculator (http://www.ual berta.ca/ stothard/javascript/prot_mw.html).

\section{Enzymatic deglycosylation}

For enzymatic deglycosylation, $20 \mu \mathrm{g}(\sim 30 \mu \mathrm{l})$ of brain or cochlea tissue lysate was mixed with or without $3 \mu$ of $10 \%$ denaturing buffer ( $5 \%$ SDS, $10 \%$ $\mu$-mercaptoethanol), boiled at $100^{\circ} \mathrm{C}$ for $10 \mathrm{~min}$, cooled, and treated with 1:10 volume of each $0.5 \mathrm{M}$ sodium phosphate buffer ( $\mathrm{pH} 7.5$ ) and 10\% NP-40. The addition of the NP-40 is to counter the effects of the SDS and ensure proper enzymatic activity (Blass et al. 1998). The mixture was then digested with 100 $\mathrm{mU}$ of Neuraminidase (New England Biolaboratories, Beverly, MA, USA) or $1000 \mathrm{U}$ of $\mathrm{N}$-glycosidase F (PNGase F; New England Biolaboratories) at $37^{\circ} \mathrm{C}$ overnight. The samples were then boiled with $2 \times$ SDS sample buffer and subjected to SDS-PAGE and Western blotting as previously described.

We used the glucose transporter-4 (Glut-4) as a positive control for our deglycosylation experiments because it is known to undergo extensive glycosylation in muscle and fat (Hresko et al. 1994). The extent of glycosylation was examined using anti-Glut4 (rabbit polyclonal, 1:1000; Calbiochem, San Diego, CA, USA) on Western blots of muscle lysates with and without enzymatic digestion. Samples were boiled with $2 \times$ SDS sample buffer and subjected to SDSPAGE and Western blotting as described above.

\section{Immunocytochemistry}

Alternating serial sets of brainstem sections were collected and preincubated in $3 \% \mathrm{H}_{2} \mathrm{O}_{2}$, followed by Tris-buffered saline (TBS, $\mathrm{pH}$ 7.6) rinses, and then preincubation in normal serum (the animal in which secondary antibodies were raised). Antisera were diluted in a solution containing $0.1 \mathrm{M}$ TBS, $1 \%$ normal serum, and $0.1 \%$ Triton X-100 (TBSNT). Brainstem sections were incubated with anti-ChT1 antiserum (1:500, AB5966 from Chemicon or a gift from H. Misawa, Department of Neurology, Tokyo Metropolitan Institute for Neuroscience, Tokyo, Japan) overnight at $4^{\circ} \mathrm{C}$ and then with an antirabbit biotinylated secondary antibody (1:200) for $2 \mathrm{~h}$. After overnight rinsing, primary and secondary antibody incubations are repeated to increase sensitivity. An avidin-biotin complex method (Vectastain Elite ABC kit; Vector Laboratories, Burlingame, CA, USA) was used for histochemical detection. Sections are first incubated with an avidin-biotin peroxidase solution and then reacted with a diaminobenzidine glucose oxidase solution $(0.025 \%$ diaminobenzidine, $1 \%$ glucose oxidase, and $0.02 \%$ glucose). Following reactions, brainstem sections were either dried and coverslipped with Crystal Clear or dehydrated, cleared in xylenes, and coverslipped with DPX (Aldrich Chemical, Milwaukee, WI, USA).

For each animal, one ear was typically used for whole organ of Corti preparations and one ear used for sectioning. We used a minimum of three animals for each age time point (except at $\mathrm{P} 0, \mathrm{P} 2$, and $\mathrm{P} 4$ where we used 6-10 animals each). In multiple labeling experiments, antisera were applied in serial tissue section sets that included one section for multiple labeling and single labeling control sections. Primary antisera were applied to a minimum of six sections per cochlea. Both organ of Corti sections and whole organs were briefly rinsed with $0.1 \mathrm{M}$ PBS for 5 min at room temperature. Samples were permeabilized with $1 \%$ Triton X-100 in $0.1 \mathrm{M}$ PBS for $1 \mathrm{~h}$ at room temperature. Samples were blocked with $10 \%$ normal goat serum (NGS) in 0.1 M PBS containing $1 \%$ Triton X-100 and then incubated with antibodies against cholinergic markers: ChT1, VAChT (antirabbit, 1:1000; Chemicon); and axon terminal markers: GAP43 (antimouse, 1:500; Sigma, St. Louis, MO), Synapsin I (antirabbit, 1:500; Chemicon), or SNAP-25 (antimouse, 1:2000; BD Transductions, Palo Alto, CA, USA). Primary antibodies were diluted in $2 \%$ NGS containing $0.05-0.1 \%$ Triton $\mathrm{X}-100$ and applied to cochlear tissues overnight at $4^{\circ} \mathrm{C}$. In certain instances, hair cell bodies were visualized by incubating cochlear tissues with antibodies against mouse $\alpha$-parvalbumin (1:1000 dilution; Sigma; clone PARV-19). Alexa 488 goat anti-rabbit secondary antibody (Molecular 
Probes, Eugene, OR, USA), Alexa 647 goat antimouse (Molecular Probes), or Cy3 donkey antimouse (Chemicon) secondary antibodies were used to visualize immunoreactivity. The specificity of primary antisera was confirmed in experiments using secondary antisera in the absence of primary antibody (data not shown).

\section{Acetylcholinesterase histochemistry}

Alternating serial sets of brainstem sections were reacted for acetylcholinesterase activity using a previously described procedure (Karnovsky and Roots 1964; Yao and Godfrey 1993). Briefly, brainstem sections are incubated overnight at $4^{\circ} \mathrm{C}$ in a buffered ( $\mathrm{pH}$ 5.6) medium containing acetylthiocholine iodide, cupric sulfate, potassium ferricyanide, and a blocker of pseudocholinesterase. Overnight incubation produced superior cellular morphology. Additionally, fewer neurons and axons were labeled with shorter incubation times. After incubation, sections were mounted on slides as described above.

\section{Imaging}

Using a laser scanning confocal microscope (BioRad, Hercules, CA, USA), fluorescently labeled whole mounts and frozen sections of the organ of Corti
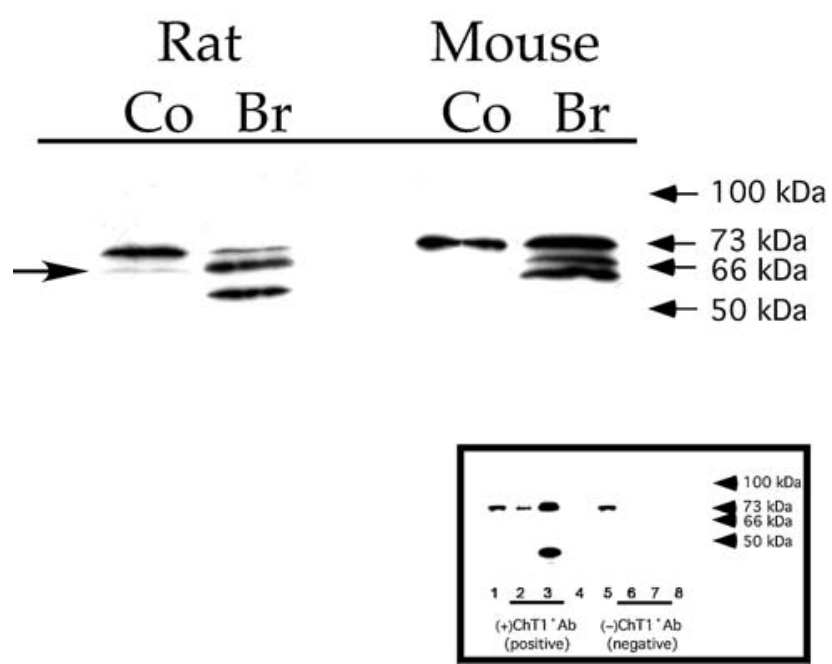

FIG. 1. Western blot of the choline transporter (ChT1) in brain and cochlear lysates. Molecular weight markers are given at 50 and 100 $\mathrm{kDa}$. The mouse P6 brain $(\mathrm{Br})$ sample shows triplet bands between 60 and $75 \mathrm{kDa}$ and the mouse P6 cochlear (Co) sample shows a single band at approximately $73 \mathrm{kDa}$. The rat $\mathrm{P} 6$ brain $(\mathrm{Br})$ sample shows a slightly variant triplet band. The rat P6 cochlear (Co) sample shows a major band and also a minor band (small arrow). Inset: Control Western blot of the sample minus primary antibody have no detectable bands. Lane 1: ladder, lane 2: mouse brain + ChT1 antibody, lane 3: mouse cochlea + ChT1 antibody, lane 4: ladder, lane 5: ladder, lane 6: mouse brain - ChT1 antibody, lane 7: mouse cochlea - ChT1 antibody, lane 8: ladder. were sequentially scanned at high (500-1000×) magnification, exciting the green, red, and far-red channels. Fluorescent emissions were separated with appropriate blocking and emission filters, scanned at slow (50 lines/s) scan speeds for high resolution, and independently detected with 8-bit accuracy by photomultiplier tubes using, if necessary, accumulation or averaging (Kalman) to increase signal and reduce noise. Identical acquisition parameters were used for all control and experimental scans. Image stacks were imported into either Volocity (v. 2 and 3; Improvision, Lexington, MA, USA) and/or ImageJ (v. 1.29×, Wayne Rasband, NIH), colorized, and merged. Three-dimensional images of serially reconstructed image stacks from the confocal microscope were rendered using Volocity. Whole mount images in the $X-Y$ plane were digitally rotated and viewed in the $X-Z$ plane. Z-projections of image stacks were performed by ImageJ. Single images were exported to GraphicConverter (Lemke Software, Germany) and image quality (brightness/contrast or histogram levels) was adjusted to maximize signal and minimize background.

\section{RESULTS}

\section{Expression of ChT1 in rodent cochlea and brain}

Polyclonal antisera raised against the $\mathrm{C}$ terminus of the high-affinity choline transporter (ChT1) were used to investigate the expression of ChT1 in the developing rodent inner ear (Misawa et al. 2001). SDS-PAGE and Western blot experiments using ChT1 antisera yielded major bands with molecular weights between 60 and $75 \mathrm{kDa}$ in brain and cochlear homogenates from postnatal day 6 (P6) animals. These bands were mostly higher in molecular weight than the expected size (approximately $64 \mathrm{kDa}$ ) predicted from the mouse ChT1 amino acid sequence (580 aa, accession number Q8BGY9). Triplet bands (two major and one minor) were identified from mouse brain homogenates but only a single band was found in mouse cochlear homogenates (Fig. 1). The single band in mouse cochlear homogenates was approximately $73 \mathrm{kDa}$ and was similar to the highest molecular weight of the mouse brain triplet bands. For comparison, Western blots of rat brain homogenates also yielded triplet bands, but they had slightly variant molecular weights and intensities from mouse brain (Fig. 1). Additionally, rat cochlear homogenates yielded a single intense band $(\sim 73 \mathrm{kDa})$ and also a very weak second band at slightly lower molecular weight (left small arrow) that is not seen in the mouse cochlear homogenates. In all cases, control blots performed without the addition of secondary antibody showed no labeling. 
Because the ChT1 on Western blot appears in our study as multiple bands between roughly 60 and 75 $\mathrm{kDa}$ except in the mouse cochlea, we hypothesized that differences in carbohydrate modification in vivo might account for our observations. To examine the nature and degree of glycosylation, homogenates of brain or cochlea from P10 mice were incubated with neuraminidase (removes sialic acid residues) or PNGase F (removes all $N$-linked sugars). The digested samples were subjected to Western blotting as described above, and results are shown in Figure 2. Enzymatic digestion did not cause the higher molecular band to disappear. However, in mouse cochlear samples, enzymatic digestion gave rise to the appearance of a doublet band. Deglycosylation with neuraminidase, and not PNGase $\mathrm{F}$, gave rise to an additional band at $45 \mathrm{kDa}$ in both mouse brain and cochlear homogenates. Deglycosylation with PNGase $\mathrm{F}$ increased the intensity of a band just below the original $73-\mathrm{kDa}$ band, similar to the doublet seen in the rat cochlea (Fig. 1). Thus, only a fraction of ChT1 appears to be extensively glycosylated in vivo, suggesting that the multiple ChT1 bands observed are attributable to other factors.

\section{ChT1 immunoreactivity in the mouse brainstem}

Brainstem sections from eight mice (two brains each at P10, P15, P20, and P50) were processed for ChT1 immunocytochemistry and AChE histochemistry. Brainstem nuclei within the superior olivary complex were classified on the basis of previous reports (Osen et al. 1984; Brown 1993; Simmons and Raji-Kubba 1993; Warr et al. 1997). As shown in Figure 3, darkly staining, ChT1-positive cell bodies are found in rostral and ventral periolivary regions of the superior olivary complex that correspond to the rostral periolivary (RPO) and ventral nucleus of the trapezoid body (VNTB), respectively, and are occasionally found near the neuropil (shell) surrounding the lateral superior olive (LSO) (Fig. 3A,C). Although no darkly staining, ChT1-positive cells were observed in the LSO or other areas of the superior olive, adjacent sections demonstrated AChE-positive cells within the LSO, LSO shell, and periolivary areas (Fig. 3B,D). Comparing adjacent ChT1 and AChE sections also leaves the impression that clusters of darkly staining ChT1-positive cells represent a subset of the AChEpositive cell labeling. As reported in previous studies, cholinergic cell bodies in rostral and ventral periolivary regions are a part of the medial OC system (Simmons et al. 1998; Vetter et al. 1991; Vetter and Mugnaini 1992). The lack of ChT1 labeling within the LSO suggests that ChT1 may be preferentially expressed by medial OC neurons.

ChT1 immunoreactivity in the mature mouse cochlea

Since medial OC axons terminate directly on the OHCs in the cochlea, we investigated whether ChT1 was restricted to an OHC innervation in the young

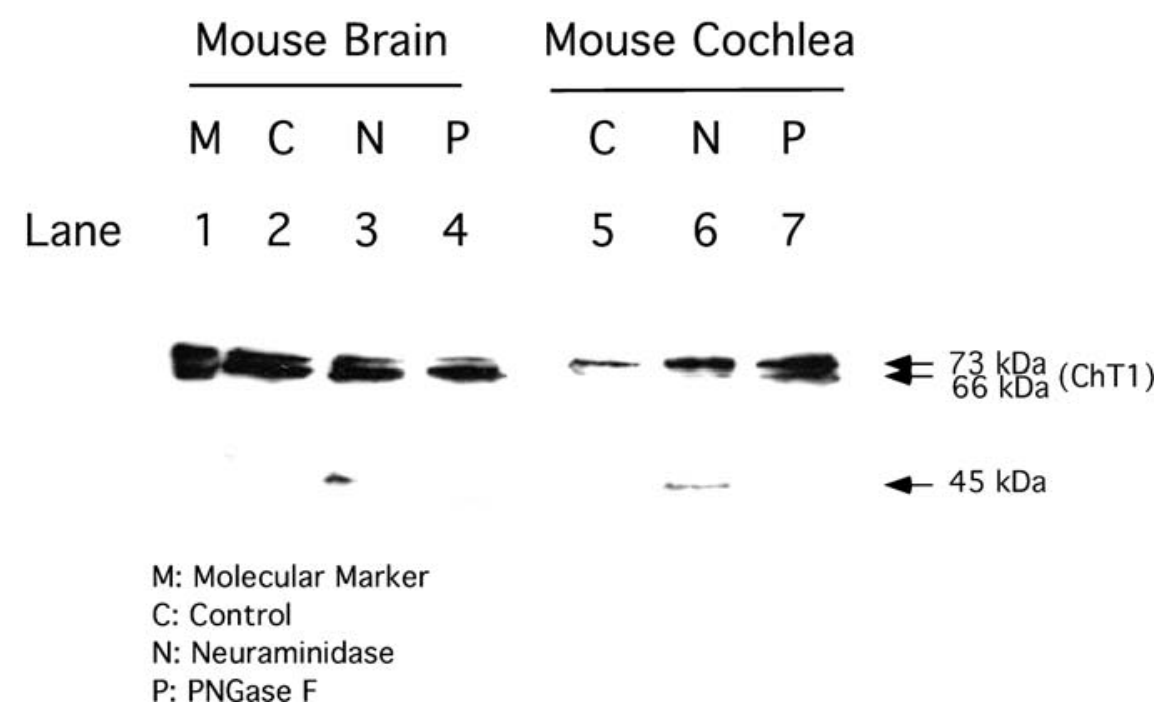

FIG. 2. Glycosylation of ChT1 protein in vivo in mouse. Total membrane lysates obtained from the P10 mouse brain and cochlea were digested with either Neuraminidase $(N)$ or PNGase $F(P)$ to remove specific carbohydrates. Samples were subjected to SDS-PAGE ( $12 \%$ acrylamide gel), and Western blot analysis was performed using anti-ChT1 antibody. M lane, molecular marker; C lanes, control sam-

ple without enzyme treatment; $\mathrm{N}$ lanes, Neuraminidase-digested sample; P lanes, PNGase F-digested sample. Note that the ChT1 immunoreactive upper band was partially deglycosylated in the brain tissue. Although not shown, control digestions in muscle lysates demonstrated a shift in the molecular weight of the glucose transporter-4 (Glut-4) as previously shown (Hresko et al. 1994). 


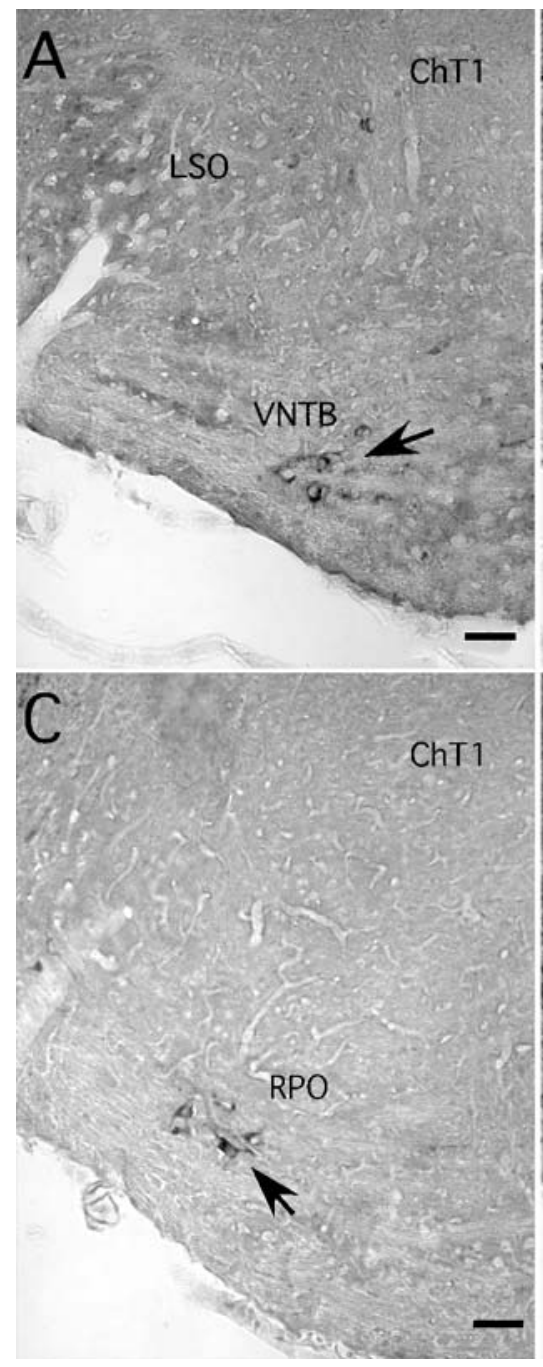

FIG. 3. Comparison of ChT1-positive and AChE-positive cells in consecutive coronal sections through the superior olivary complex of a P20 mouse brain. (A) A small cluster of ChT1-positive cells (arrow) labeled intensely in the ventral nucleus of the trapezoid body (VNTB). The lateral superior olive (LSO) had a few weakly labeled cell bodies in its lateral limb. Lateral (L) and dorsal (D) orientations are as iden-

adult mouse cochlea. As shown in Figure 4A, ChT1 immunoreactivity (ChT1-ir) in the P20 mouse cochlea was almost exclusively localized below all three rows of OHCs. To demonstrate that ChT1-ir labeled cholinergic efferent terminals, synapsin Ia immunoreactivity (synapsin-ir) and vesicular acetylcholine transporter immunoreactivity (VAChT-ir) were compared to ChT1-ir. Adjacent serial sections demonstrated that synapsin-ir and VAChT-ir were both present below IHCs and OHCs (Fig. 4B,C). In contrast, ChT1-ir was barely detected below IHCs where there was light labeling in the inner spiral bundle (ISB) region. Below OHCs, the pattern of ChT1-positive terminals was very similar to synapsin-

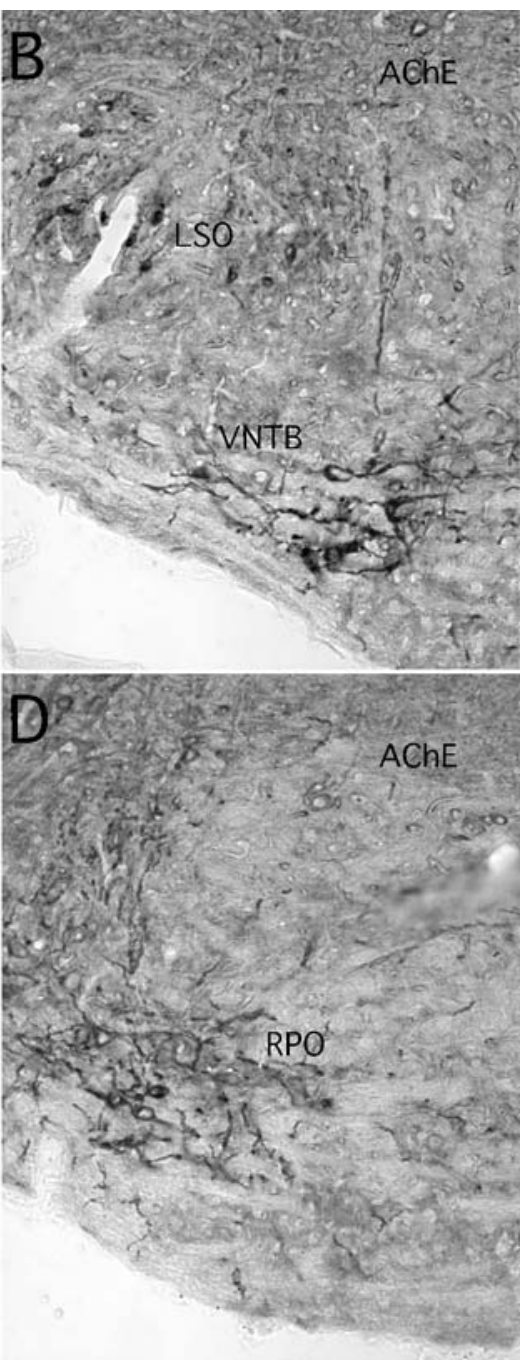

tified. The scale bar represents $50 \mu \mathrm{m}$. (B) AChE-positive cells are found in the VNTB and LSO. The scale bar is the same as in panel A. (C) ChT1-positive cells (arrow) labeled intensely in the rostral periolivary (RPO) region. The scale bar represents $50 \mu \mathrm{m}$. (D) AChEpositive cells stained in the RPO. The scale bar is the same as in panel C.

and VAChT-positive terminals. The observed labeling patterns is consistent with synapsin-ir and VAChT-ir labeling medial and lateral OC terminals, whereas ChT1-ir labels mostly, if not exclusively, medial OC terminals.

To confirm that our ChT1 results were not age- or strain-specific to ICR mice, we compared ChT1 labeling in older P50 ICR and P180 C57BL cochleae. In the older ICR cochlea (P50), there was still a lack of any substantial ChT1-ir below the IHCs although synapsin-ir and VAChT-ir were present (Fig. 4D-F). At least in the midbasal cochlea, there was also little, if any, difference between ChT1-, synapsin-, and VAChT-ir in OHC terminals. Even in C57BL mice at 
ChT1
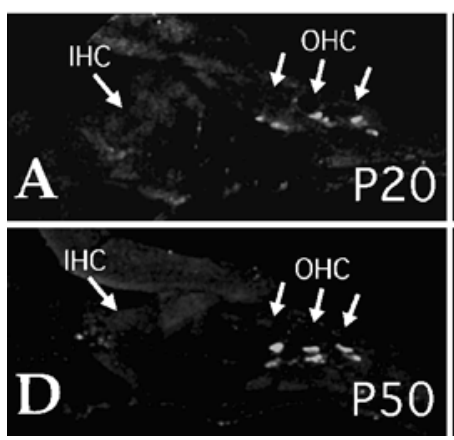

Synapsin
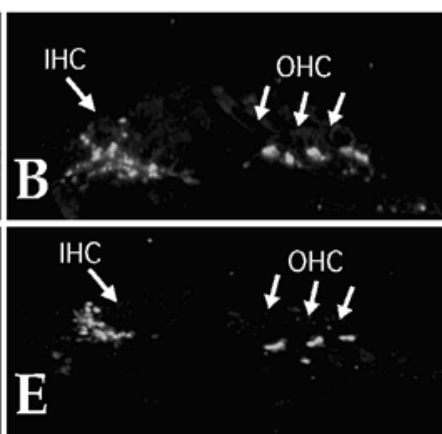

VAChT

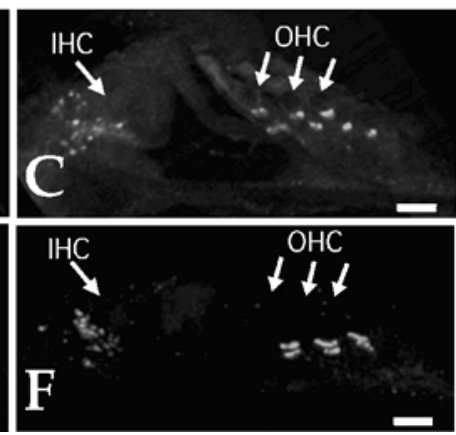

FIG. 4. Confocal micrographs of ChT1-ir, synapsin-ir, and VAChT-ir on consecutive midmodiolar sections of midbasal regions of mouse cochlea. In the P20 (A-C) and P50 (D-F) mouse cochlea, ChT1-ir (A, D) was almost exclusively localized below $\mathrm{OHCs}$, whereas synapsin-

P180, only weak ChT1-ir was visible in the ISB region below IHCs, whereas robust ChT1-ir was visible below OHCs (data not shown).

ChT1 immunoreactivity in the developing mouse cochlea

Our previous studies suggest an apparent temporal order to cholinergic differentiation within OC neurons that coincides with peripheral innervation (reviewed in Simmons 2002). Such observations led us to hypothesize that medial OC efferent axons may be present prior to the completion of cholinergic differentiation. Although apical through basal regions of the cochlear spiral were always examined, we limit our developmental study as we have done previously to the middle turn (midbasal region, i.e., roughly middle third) of the cochlea across age points. To label growing, immature efferent axons, we used an antiserum against growth-associated protein 43 (GAP43) and compared GAP43 innervation patterns to the expression of ChT1 in midbasal regions of the mouse cochlea during embryonic (E18) and early postnatal (P0-P4) periods of development. During these ages, GAP43 immunoreactivity (GAP43-ir) was restricted to regions of the cochlea consistent with the projections of efferent OC axons. As shown in Figure 5, GAP43-ir was observed in the intraganglionic spiral bundle (IGSB), within a subset of axons in the osseous spiral lamina (OSL), and was initially restricted to the IHC region of the E18 and P0 cochlea (Fig. 5A,B). No GAP43-ir was detected within cell bodies or axons of spiral ganglion neurons, the neuronal population that gives rise to the afferent innervation of the cochlea (Fig. 5A,B). These observations are consistent with the view that GAP43 labels mostly, if not exclusively, efferent OC ir (B, E) and VAChT-ir (C, F) were visible below both IHCs and OHCs. The weak ChT1-ir seen below IHCs is in the region of the ISB. Scale bar $=10 \mu \mathrm{m}$.
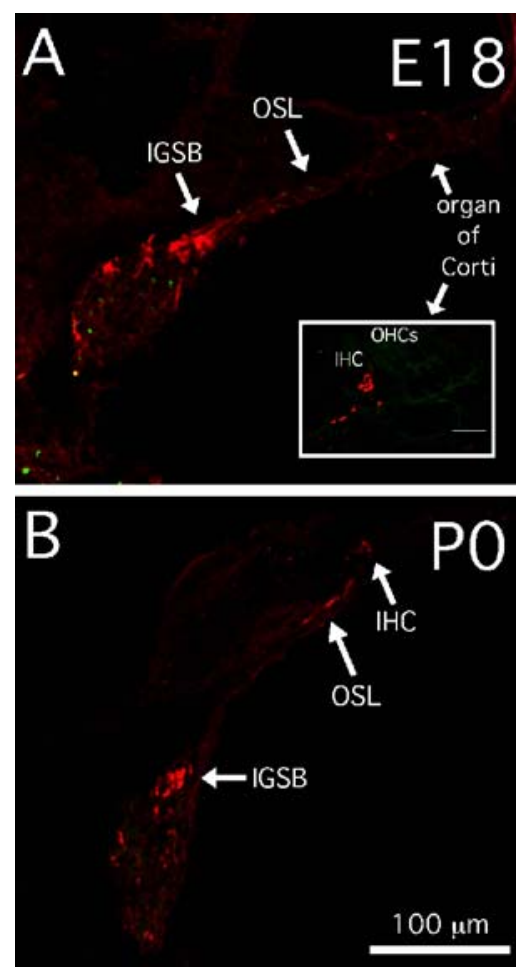

FIG. 5. Confocal micrographs of GAP43-ir and ChT1-ir on midmodiolar sections of midbasal regions of the mouse cochlea at E18 (A) and P0 (B). In A and B, GAP43-ir (red) is visible in the intraganglionic spiral bundle (IGSB) and the osseous spiral lamina (OSL) as well as below inner hair cells (IHC). GAP43-ir was not visible in the cell bodies of spiral ganglion neurons, indicating that GAP43 preferentially labels efferent neurons in the mouse cochlea. ChT1 (green) was not visible in the IGSB, OSL or below IHCs at E18 or $\mathrm{P} 0$ in mouse cochlea. The inset in panel A shows a higher magnification of the organ of Corti at E18 with GAP43-ir (red) below IHCs but not below outer hair cells (OHCs). In the inset, the green channel was enhanced to demonstrate the lack of any ChT1-ir. Scale bar $=10 \mu \mathrm{m}$. 
GAP43

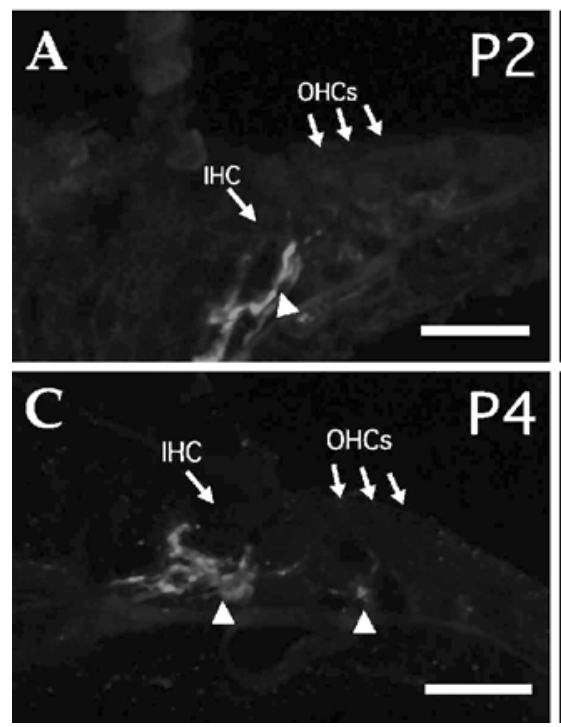

FIG. 6. Z-projections of confocal micrographs show GAP43-ir (A, C) and ChT1-ir $(B, D)$ on midmodiolar sections of midbasal regions of mouse cochlea at P2 (A, B) and P4 (C, D). (A) GAP43-ir is present below IHCs at P2. (B) ChT1 is not visible below IHCs or OHCs at P2.

axons in the late embryonic and early postnatal mouse cochlea (Merchan-Perez et al. 1993).

In midbasal regions of the $\mathrm{E} 18$ and $\mathrm{P} 0$ mouse cochlea, ChT1-ir was not visible below IHCs, nor was it visible in the IGSB (Fig. 5A,B). At P2, GAP43-ir was present beneath IHCs and in the region of the presumptive ISB, whereas ChT1-ir was typically not detected below IHCs (Fig. 6A,B). Only one out of 10
ChT1

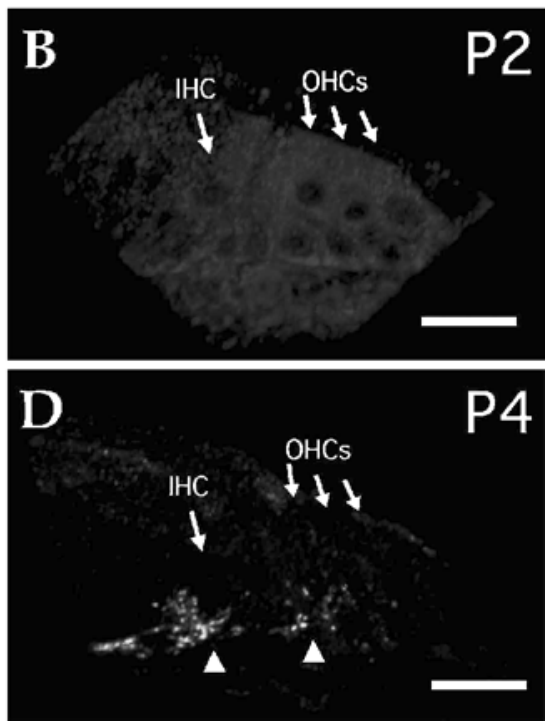

(C) GAP43-ir is present below both IHCs and OHCs at P4. (D) ChT1-ir is visible below IHCs, between inner and outer pillar cells, and a few ChT1-positive nerve terminals were scattered below the OHCs at P4.

P2 animals investigated had any (just detectable) ChT1-ir below IHCs and this labeling was restricted to midbasal regions of the cochlea (see Fig. 7A). The variable ChT1 labeling at $\mathrm{P} 2$ suggests that this age is the beginning of ChT1 expression. By P4, GAP43-ir was observed at the basolateral surface of IHCs, between both the inner and outer pillar cells, and terminated below at least the first row of OHCs

\section{ChT1}
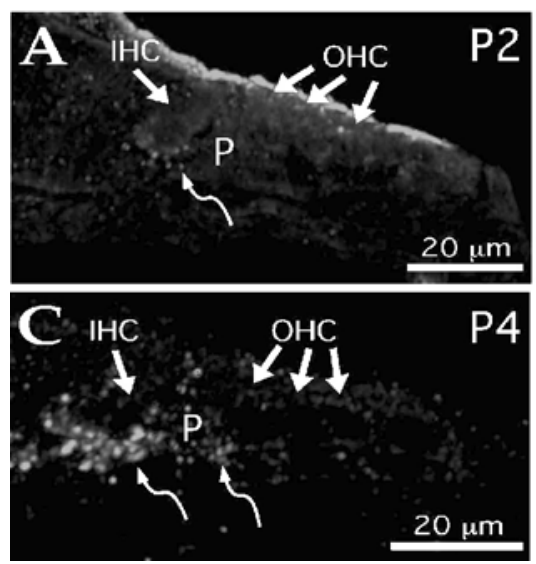

FIG. 7. Confocal micrographs of ChT1-ir (A, C) and VAChT-ir (B, D) on serial midmodiolar sections of midbasal regions of mouse cochlea at P2 and P4. (A) ChT1-ir (curved arrow) was just detectable below IHCs. This animal was the only example $(n=10)$ of ChT1-ir below IHCs at P2. (B) VAChT-ir (curved arrow) was clearly present below IHCs. (C) ChT1-ir (curved arrows) was visible below IHCs, between

\section{VAChT}
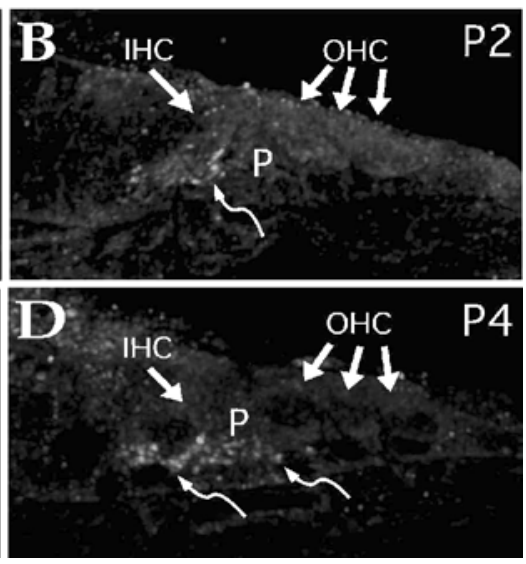

inner and outer pillar cells (P), and a few ChT1-positive terminals were visible below the first row of OHCs. (D) VAChT-ir (curved arrows) was visible below IHCs, between pillar cells, and below the first row of OHCs. Scale bars are the same for each serial set (A-B, C$\mathrm{D}$, and $\mathrm{E}-\mathrm{F})$. 
(Fig. 6C). Similarly, ChT1-ir consistently surrounded the base of IHCs and was between inner and outer pillar cells, and a few ChT1-positive nerve terminals were scattered below the first row of OHCs (Fig. 6D). Significantly at $\mathrm{P} 4$, all animals $(N=6)$ showed ChT1ir below IHCs, but not all animals showed ChT1 labeling below OHCs (two out of six animals). Although the majority of GAP43-ir and ChT1-ir did not colocalize at P4, a small amount of overlap was visible on the inner pillar side of the IHC (data not shown). The somewhat variable labeling of ChT1 at ages P2 and P4 across animals suggests that these ages may be transitional for the beginning of ChT1 expression below IHCs and OHCs, respectively.

To verify that ChT1 labels cholinergic efferent neurons in the developing ear, we compared ChT1-ir to the expression of VAChT (Fig. 7). Similar to GAP43-ir around birth, VAChT-ir was restricted to the IGSB and OSL. At P0, VAChT-ir was present below IHCs in nine out of 10 animals examined. In two P2 animals, one ear was processed for VAChT and the other was processed for ChT1; in both cases, VAChT-ir was established below IHCs except for apical portions of the cochlea whereas ChT1-ir was not detected anywhere along the cochlear spiral. Figure 7A,B show the only case where ChT1-ir was just detectable at P2 and VAChT-ir was visible below IHCs in every mouse examined. Comparison of adjacent ChT1 and VAChT labeled sections from a P4 mouse cochlea show that VAChT-ir was visible below IHCs and the first row of OHCs, whereas ChT1 was restricted to the IHC region (Fig. 7C,D). Such serial section analysis of the early postnatal mouse cochlea suggests that although ChT1-ir possibly lags behind VAChT-ir, there was otherwise little, if any, difference in their immunostaining patterns. These data (e.g., Figs. 4 and 7) indicate that ChT1-ir is not only consistent with a cholinergic innervation within the cochlea, but may also be preferentially associated with medial OC axons.

Previous electron microscopic observations suggest that discernible efferent synapses are present below both IHCs and OHCs by P6 in the mouse (Emmerling and Sobkowicz 1990). However, at this stage of cochlear development, GAP43 is not useful as an OHC efferent marker because its expression is reduced. Therefore, we used antisera against SNARE complex proteins to label efferent synapses. Synapsin-ir is initially expressed below IHCs and begins between P0 and P2 in the mouse cochlea. The early expression of synapsin-ir below IHCs prior to any ChT1-ir may suggest that ChT1 is expressed following
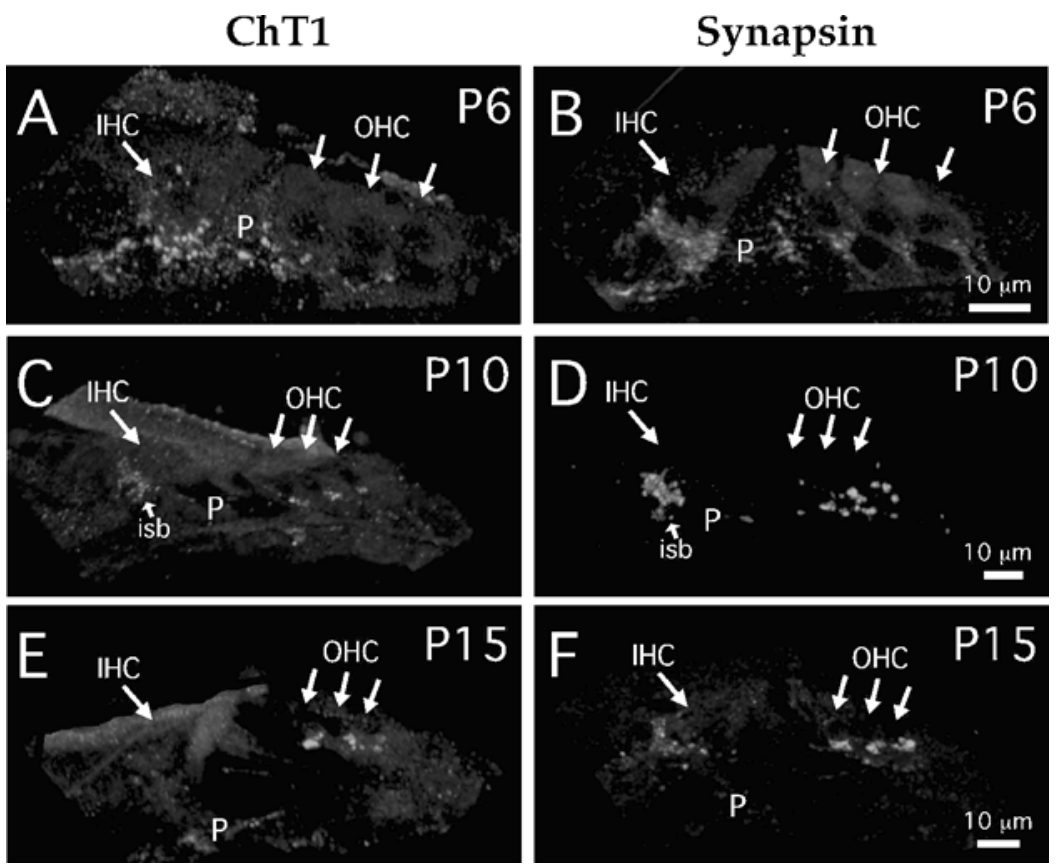

FIG. 8. Confocal micrographs of ChT1-ir (A, C, E) and synapsin-ir (B, $D, F)$ on serial midmodiolar sections of midbasal regions of mouse cochlea at P6 (A, B), P10 (C, D), and P15 (E, F). (A) ChT1-ir is visible below IHCs, between pillar cells (P), and below the first two rows of OHCs. (B) synapsin-ir is visible below IHCs, between pillar cells, and below all three rows of OHCs. (C, D) ChT1-ir and synapsin-ir have

nearly identical innervation patterns. Both ChT1-ir and synapsin-ir are visible below IHCs in the ISB, between pillar cells, and below all three rows of OHCs. Additionally, ChT1-ir appears as a subset of synapsin-ir below IHCs. (E, F) ChT1-ir is almost exclusively localized below OHCs. In contrast, synapsin-ir is visible below IHCs, in the ISB, and below all three rows of $\mathrm{OHCs}$. 
efferent synapse formation. Neither synapsin-ir nor ChT1-ir was visible within the IGSB or the OSL, indicating that the expression of $\mathrm{ChT} 1$ and synapsin Ia was restricted to efferent axon terminals.

From P6 through P15 in the mouse cochlea, ChT1ir was visible within the ISB, between inner and outer pillar cells, and below OHCs in midbasal regions of the cochlea. At P6, ChT1-ir was observed between the inner and outer pillar cells as well as below the first two rows of OHCs (Fig. 8A). In contrast, not only was synapsin-ir visible between inner and outer pillar cells, but synapsin-ir was also present below all three rows of OHCs, suggesting that synapsin-positive terminals precede the appearance of ChT1-positive terminals (Fig. 8B). Such a scenario would support the idea that ChT1 expression follows synaptic contact in the cochlea. By P10, ChT1-ir was noticeably reduced directly beneath IHCs and was present in the area of the ISB. Robust ChT1-ir was pre-sent below all three rows of OHCs without any obvious preference for the first row of OHCs at this age (Fig. 8C). At P10, synapsin-ir was visible below IHCs as well as below all three rows of OHCs (Fig. 8D). By P15, ChT1-ir was almost exclusively localized below OHCs
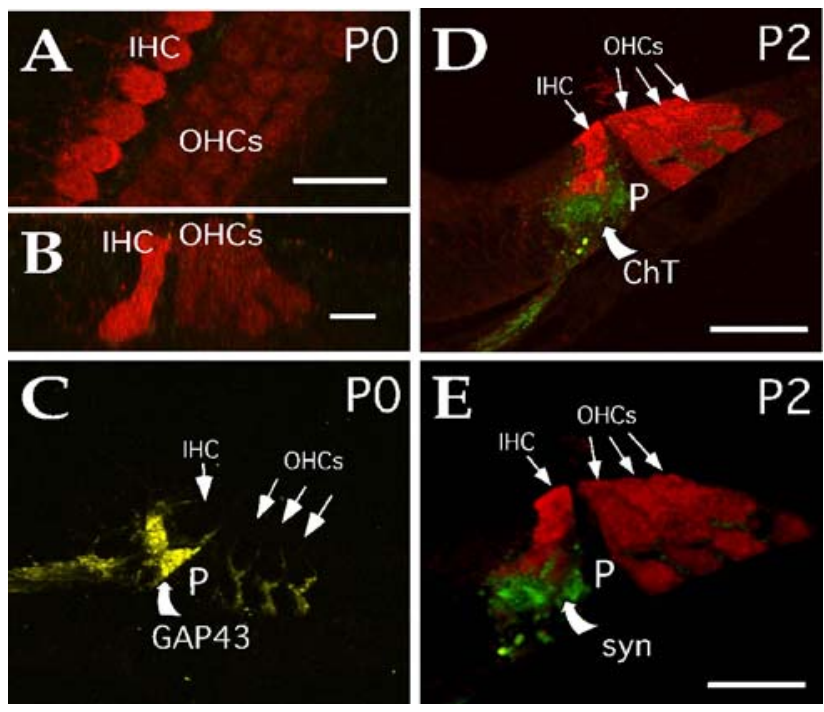

FIG. 9. The early postnatal expression of ChT1-ir and GAP43 in the rat cochlea. The inner and outer hair cells are labeled with $\alpha$ parvalbumin (red) in panels A, B, D, and E. (A) At P0, a wholemount view of the midbasal organ of Corti shows no ChT1-ir (green) near IHCs. (B) Image stacks from panel A were digitally rotated to give a cross-sectional view. No ChT1-ir was detected below IHCs. (C) In the PO rat cochlea, GAP43-ir (yellow) shows extensive labeling below the IHCs and some labeling below all three rows of OHCs. (D) At P2, a cross-sectional view of the midbasal organ of Corti shows ChT1-ir (green) completely surrounding the lower portion of the $\mathrm{IHC}$ and extending into the pillar cell region. (E) Also at P2, a cross-sectional view of the midbasal organ of Corti shows synapsin-ir below IHCs. Scale bars $=25 \mu \mathrm{m}$ in A, C, D and $10 \mu \mathrm{m}$ in B. P, pillar cell; syn, synapsin.
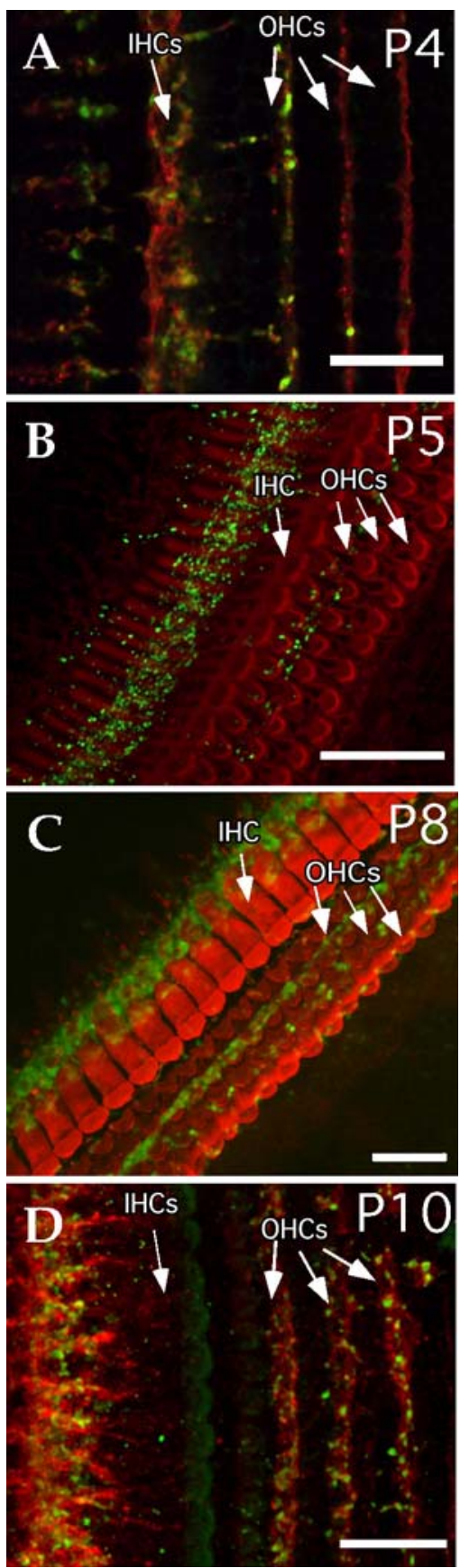

FIG. 10. Confocal images of ChT1, SNAP25, and synapsin immunoreactivities in the rat cochlea. (A) At P4, a longitudinal view of the midbasal organ of Corti shows ChT1-ir (green) and SNAP25-ir (red). (B) At P5, a whole-mount reconstruction of the midbasal organ of Corti shows synapsin-ir (green) completely surrounding the lower portion of the $\mathrm{IHC}$ and extending up to $\mathrm{OHC}$ row 2. Inner and outer hair cells are labeled for $\alpha$ parvalbumin. (C) At P8, a whole mount reconstruction of the midbasal organ of Corti shows ChT1-ir (green) surrounding the bases of IHCs and in all three rows of OHCs. (D) In a P10 longitudinal view, both ChT1-ir (green) and SNAP25-ir show labeling below the IHCs and bouton-like labeling below all three rows of OHCs. Scale bar $=25 \mu \mathrm{m}$. 

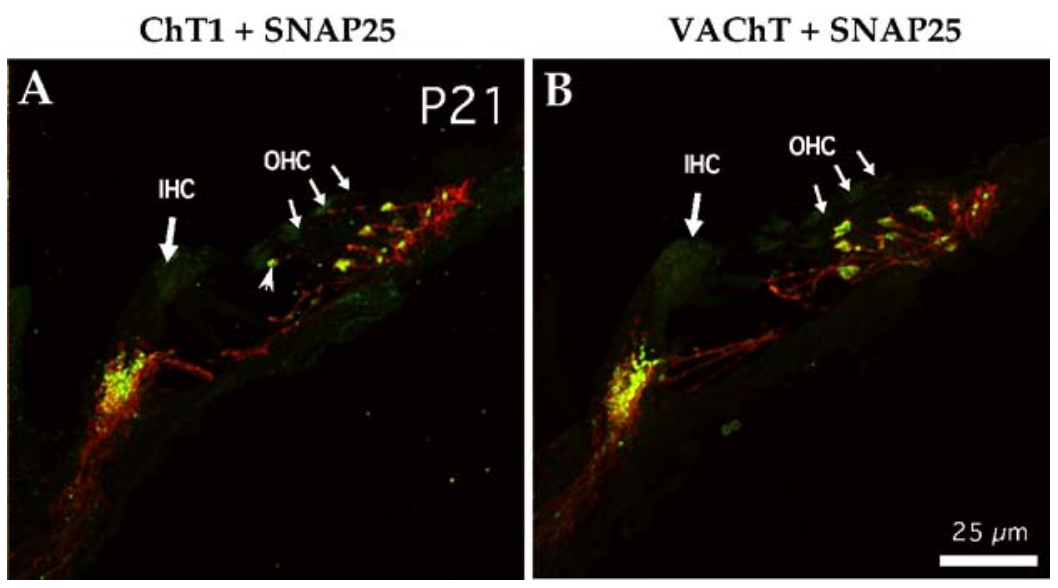

FIG. 11. Z-projections of confocal micrographs of SNAP25-ir (red) double-labeled with either ChT1-ir (A) or VAChT-ir (B) on midmodiolar sections of midbasal regions of the rat cochlea at P21. (A) ChT1-ir (green) colocalizes with most but not all of the SNAP25-ir label below IHCs and colabels virtually all of the SNAP25-ir below

OHCs. (B) VAChT-ir (green) appears identical to the ChT1-ir and colocalizes with most but not all of the SNAP-ir label below IHCs and colabels virtually all of the SNAP25-ir below OHCs. The scale is the same in A and B.

(Fig. 8E), whereas synapsin-ir was visible beneath both IHCs and all three rows of OHCs (Fig. 8F).

ChT1 immunoreactivity in the developing rat cochlea

To test the generality of ChT1 labeling being restricted to OHC efferent terminals, we did a developmental study of ChT1 expression in the rat cochlea. The rat and mouse ChT1 amino acid sequences share greater than $98.5 \%$ homology. Overall, the rat and mouse demonstrated similar patterns of ChT1 development. In the following, we

shall emphasize the major differences between rat and mouse.

The most obvious difference between mouse and rat was that the rat showed an earlier postnatal expression of ChT1 than the mouse. At P0, there was no detectable ChT1-ir in the organ of Corti (Fig. 9A,B). However, similar to the mouse cochlea, GAP43-ir was restricted to the IGSB and present below inner and outer hair cells (Fig. 9C). In all P2 rats $(N=4)$, ChT1-ir (Fig. 7D) and synapsin-ir (Fig. $7 \mathrm{E})$ were present below IHCs with ChT1-ir positive terminals also observed among pillar cells (Fig. 9D). The earlier postnatal appearance of ChT1 in the rat compared to mouse is consistent with the differences

TABLE 1

\begin{tabular}{|c|c|c|c|c|c|c|}
\hline \multirow[b]{4}{*}{ Age } & \multicolumn{6}{|c|}{ Summary of cholinergic labeling of efferent terminals on hair cells in the mouse and rat cochlea } \\
\hline & \multicolumn{4}{|c|}{ Mouse } & \multirow{2}{*}{\multicolumn{2}{|c|}{$\begin{array}{c}\text { Rat } \\
\text { ChT1 }\end{array}$}} \\
\hline & \multicolumn{2}{|c|}{ VAChT } & \multicolumn{2}{|c|}{ ChT1 } & & \\
\hline & IHC & $\mathrm{OHC}$ & IHC & $\mathrm{OHC}$ & IHC & $\mathrm{OHC}$ \\
\hline E18 & - & - & - & - & - & - \\
\hline PO & + & - & - & - & \pm & - \\
\hline P2 & ++ & - & \pm & - & ++ & \pm \\
\hline P4 & ++ & + & +++ & \pm & +++ & + \\
\hline P6 & & & +++ & ++ & +++ & ++ \\
\hline P10 & ++ & +++ & ++ & ++ & +++ & ++ \\
\hline P15 & ++ & +++ & \pm & +++ & +++ & +++ \\
\hline P20/21 & ++ & +++ & - & +++ & +++ & +++ \\
\hline Adult & & & - & +++ & +++ & +++ \\
\hline
\end{tabular}

Relative immunoreactivity quality indicated: absent $(-)$, rare or occasional $( \pm)$, weak $(+)$, moderate $(++)$, and strong $(+++)$. Rare or occasional $( \pm)$ labeling indicates faint (just detectable) immunostaining in at most one out of three animals. Weak (+) labeling indicates at least faint immunostaining in most (9 out of 10$)$ cases. Moderate $(++)$ labeling indicates medium intensity immunostaining that is well delineated in all animals. Strong (+++) labeling indicates intense immunoreactivity in all animals. 
in gestational ages between the two species (Simmons 2002).

In the rat cochlea between $\mathrm{P} 4$ and P10, ChT1-ir followed a similar progression as found in the mouse between P6 and P15, except that ChT1 did not diminish below the IHCs. At P4 and P10, rat cochleae were double-labeled for ChT1-ir and SNAP25 immunoreactivity (SNAP25-ir), another marker specific for presynaptic axon terminals. At $\mathrm{P} 4$ and P5, ChT1-ir and SNAP25-ir were found mostly below IHCs with some extensions to the first and second rows of OHCs (Fig. 10A,B). There was a degree of ChT1-ir and SNAP25-ir colocalization below IHCs and on the first row of OHCs (Fig. 10A). By P8, ChT1-ir was present below IHCs and all three rows of OHCs (Fig. 10C). At P10, ChT1-ir extensively colocalized with SNAP25-ir below IHCs and all three rows of OHCs (Fig. 10D). However, unlike in the mouse cochlea, ChT1-ir was not diminished below the IHCs.

Double labeling experiments at P21 in the rat cochlea show that ChT1-ir colocalizes extensively with SNAP25-ir (Fig. 11A) similar to the manner in which VAChT-ir colocalizes with SNAP25-ir (Fig. 11B). Both ChT1 and VAChT colocalize with SNAP25 below IHCs and all three rows of OHCs. Below both IHCs and OHCs, ChT1-ir and VAChT-ir appear to be a subset of SNAP25-ir and were virtually indistinguishable from one another.

\section{DISCUSSION}

Our results indicate that the high-affinity choline transporter is associated with cochlear efferent innervation, is most likely expressed after immature efferent axons arrive below IHCs, and may be differentially expressed within subpopulations of OC neurons in some species. Efferent OC axons arrive within the primordial cochlear epithelium as early as E12 in the mouse (Fritzsch and Nichols 1993) and are found in the vicinity of IHCs as early as E17E18 in the mouse (Sobkowicz and Emmerling 1989) and by about $\mathrm{P} 0$ in the rat (Bruce et al. 2000). Unlike GAP43-ir, which labels growing efferent axons, ChT1ir was not present in the embryonic or the early postnatal mouse (prior to P2) or rat (prior to P0) cochlea. Although somewhat variable, the onset of ChT1 expression was typically at P2-P4 in the mouse cochlea and $\mathrm{P} 0-\mathrm{P} 2$ in the rat cochlea. In comparison, other cholinergic markers such as VAChT or presynaptic markers such as synapsin were always present prior to ChT1 detection in both mouse and rat. Table 1 summarizes the temporal expression of ChT1-ir in the mouse and rat. The consistent differences in the temporal expression patterns of ChT1 and VAChT suggest that the induction of these two cholinergic proteins may be either coincident with or immediately following the arrival of efferent axon terminals to the IHC area.

Other studies report that AChE and ChAT activities can be measured at birth in the mouse cochlea, and that these levels increase sharply during the first 10 days of postnatal development (Sobkowicz and Emmerling 1989). Such studies support the view that mature levels of cholinergic expression and cholinergic differentiation follow the arrival of efferent OC axons in the cochlea. Thus, the temporal separation between the arrival of efferent axons and the appearance of ChT1-ir and VAChT-ir in the mouse cochlea raises the possibility that direct or indirect contact between efferent OC neurons and hair cells may be important for the induction and/or regulation of a mature cholinergic phenotype. Eliminating any contact with inner hair cells and investigating the effects on ChT1 or VAChT expression would test such a scenario, in part. An alternative possibility is that cholinergic differentiation in OC neurons occurs via a target-independent mechanism that does not require contact with hair cells. In either case, our findings are consistent with the idea that efferent axons arrive below inner hair cells, and undergo some further differentiation, such as ChT1 expression, prior to contacting OHCs. We have previously termed this period of differentiation below IHCs a developmental waiting period, which occurs prior to OHCs efferent terminations (Simmons et al. 1996a; Simmons 2002).

\section{Temporal pattern of cholinergic differentiation}

Our findings lead to the hypothesis that ChT1 may be part of the final stage of cholinergic presynaptic differentiation. There have been no systematic studies on the temporal patterns of cholinergic differentiation in the mammalian cochlea and little is known about the temporal order elsewhere in the nervous system. In our previous studies of OC neurons in the brainstem, we deduced that ChAT onset occurred first, closely followed by AChE and VAChT (reviewed in Simmons 2002). Weak ChAT immunoreactivity (ChAT-ir) has been observed below IHCs at P0 in the rat cochlea (Merchan-Perez et al. 1994); however, these investigators did not look for ChAT-ir earlier in development. Assuming a difference of roughly two days between the gestation periods of mice and rats (approximately 19.5 days for mice and 21.5 days for rats, respectively), a $\mathrm{P} 0$ rat should be the equivalent of a P2-P3 mouse. Because we found that the onset of ChT1 expression was $\mathrm{P} 0-\mathrm{P} 2$ in rats and $\mathrm{P} 2-\mathrm{P} 4$ in mice, we would expect ChAT-ir in the cochlea to precede ChT1-ir by at least three to four days (i.e., at 
least by E18). In rats, we would expect ChAT-ir in the cochlea to be present also around E18 followed by ChT1-ir three to four days later (i.e., P0-P2).

Using anterograde labeling of the crossed OC bundle, Fritzsch and Nichols (1993) and Bruce et al. (1997) were able to visualize DiI-labeled efferents in the vicinity of the growing cochlear duct at E12-13 (Fritzsch and Nichols 1993; Bruce et al. 1997). Labeled fibers started to form the IGSB in basal and middle turns by E13.5-E14.5 (Bruce et al. 1997). By E16.5, labeled fibers branched extensively among supporting cells of the greater epithelial ridge (GER) and were present among IHCs. At E18.5, efferents began to form the ISB below IHCs in basal, but not apical, regions of the cochlea (Bruce et al. 1997). In support of these observations, Sobkowicz and Emmerling (1989) reported that a few acetylcholinesterase-stained fibers (presumably efferent) were visible in the developing ISB at E17-E18. It is not clear when or if labeled efferents contact IHCs before E18 in these studies. To further evaluate the extent of efferent innervation in the mouse cochlea before birth, we labeled growing, immature efferent axons with a monoclonal antiserum against GAP43. These data indicate that GAP43-ir is restricted to the IGSB and a subset of axons in the OSL. Consistent with an efferent label, neither spiral ganglion cell bodies nor their axons have any observable GAP43-ir. Our observations indicate that GAP43-labeled axons project below, and may make contact with, IHCs as early as E18 in the mouse. Our observations are consistent with previous reports that GAP43 labels efferent OC axons in the late embryonic and early postnatal rodent cochlea (Merchan-Perez et al. 1993; Simmons et al. 1996b).

\section{Is ChT1 selective for medial OC neurons in mice?}

In adult animals, the virtual absence of ChT1 expression below IHCs would be expected if $\mathrm{ChT} 1$ were preferentially expressed by medial OC axons but not by lateral OC axons. Alternatively, it is possible that the early ChT1-positive axon terminals seen below IHCs are from both medial and lateral OC axons, but at later stages of cochlear development, ChT1 is downregulated in lateral OC neurons resulting in the exclusive localization of ChT1 below OHCs. Our examination of ChT1-ir in the mouse brainstem revealed very little expression in regions identified with lateral OC neurons; instead, ChT1-ir was in rostral and ventral periolivary regions consistent with medial OC neurons. Our results also suggest that the lack of any significant detection of ChT1 expression below IHCs in mice was not attributable to strain differences or a developmental delay. Because there was some faint ChT1 labeling, especially in LSO shell neurons, it leaves open the possibility that ChT1 is being expressed by at least some lateral neurons at low levels.

The lack of a robust expression of ChT1 in mouse lateral efferent neurons is somewhat surprising, because lateral efferent neurons demonstrate robust expression of other cholinergic enzymes and proteins such as ChAT, AChE, and VAChT. Rapid synaptic cholinergic transmission between neurons and their postsynaptic targets more than likely requires high-affinity choline transport for the synthesis of acetylcholine. The lack of any substantial ChT1-ir in lateral OC neurons in the mouse suggests that they do not utilize rapid synaptic cholinergic transmission, acquire choline from one or more alternative sources, or require much lower levels of the transporter. The choline required for lateral efferent acetylcholine synthesis could be supplied from membrane phosphotidylcholine via phospholipases; however, the role of this pathway in acetylcholine synthesis remains unclear (Tucek 1985; Okuda and Haga 2003). It is also possible that the size and/or density of lateral efferent cell bodies and synapses make it difficult to resolve ChT1-ir in cochlear sections using fluorescent microscopy or in brainstem sections using peroxidase histochemistry. Thus in the mouse, the ChT1 protein could be expressed in both medial and lateral OC neurons, but at levels in lateral OC neurons that escape detection in our experiments.

However, our results in the rat give some pause to any interpretation of the mouse ChT1 data. In general, the rat and mouse data consistently differed by roughly two postnatal days in accord with the twoto three-day gestational differences between the two species. Thus, ChT1 expression below IHCs was present at $\mathrm{P} 2$ in rats compared to $\mathrm{P} 4$ in mice, and below OHCs at $\mathrm{P} 4$ in rats and $\mathrm{P} 6$ in mice. This two- to three-day maturation differential was also seen for GAP43, synapsin, and VAChT expression. However, a striking difference in ChT1 expression occurred between rats and mice in the second postnatal week, where ChT1 expression below IHCs remained high instead of disappearing. This expression of ChT1 below IHCs in the rat suggests that both lateral and medial OC axons express ChT1 over the course of postnatal development. These data raise again the specter of different ChT1 isoforms being expressed by lateral OC axons in the mice or other differences in high-affinity uptake mechanisms as mentioned above.

Could the difference in ChT1 expression below IHCs in the mouse and rat cochlea be attributable to the different isoforms of ChT1 that either are not recognized or weakly recognized by the antisera used in these investigations? The amino acid sequences of 
rat and mouse ChT1 are nearly 99\% homologous and no known isoforms have been suggested to date. In the C-terminal region from which the antisera were made, there is also about 99\% sequence identity between rat and mouse ChT1. Other studies using ChT1 antisera generated from the C-terminal region identify a broad distribution of protein bands ranging in size from 60 to $75 \mathrm{kDa}$ (Okuda et al. 2000; Ferguson et al. 2003; Hoover et al. 2004). Our Western blot results on mouse and rat brain and cochlear tissue homogenates suggest that the ChT1 antisera recognize multiple bands that are all higher in molecular weight than the predicted size. Therefore, to explain our observations, it is possible that the ChT1 antisera recognizes either a region of the ChT1 protein that is poorly conserved between rats and mice or that this antibody might bind to a carbohydrate modification that is unique in the rat (i.e., a band that is present in the rat but not in the mouse) in addition to binding the ChT1 glycoform shared by both rat and mouse.

Does the difference between mouse and rat ChT1 represent variations in glycoforms? Glycosylation is one of the most abundant post- and cotranslational modifications of proteins in mammals. Variations in the glycan moieties of glycoproteins lead to different glycoforms of the protein and affect protein activity and function. Both mouse and rat ChT1 amino acid sequences are predicted to have the same three $N$ linked glycosylation sites using the on-line database of proteins and domains (http://www.expasy.org/ prosite). Ferguson et al. (2003) report that ChT1 produced in transfected PC12 cells is a $65-\mathrm{kDa}$ glycoprotein that shifts to $45 \mathrm{kDa}$ after deglycosylation with PNGase F. Because the ChT1 bands in brain homogenates appear as a triplet and yet contain the same peptide sequences, the differences in carbohydrate modification might account for the differences between bands. However, our deglycosylation studies with neuramidase and PNGase F in tissue homogenates indicate otherwise. Although we did find a band at $45 \mathrm{kDa}$ after neuramidase treatment, the higher molecular weight bands were still present. The difference in results could be attributable to the use of different antibodies. Alternatively, there could be a substantial fraction of the ChT1 protein that is not glycosylated in vivo in rodents.

In conclusion, our observations suggest that highaffinity choline transport is involved in the synthesis of acetylcholine in efferent OC nerve terminals. During mammalian cochlear development, ChT1 is detected after efferent axon terminals have arrived below IHCs and after VAChT expression, indicating that high-affinity choline transport may be the final stage of cholinergic differentiation in OC neurons. Finally, our data indicate that there may be species differences in the expression of ChT1 within the OC system. These results raise many potentially interesting questions that require much further study.

\section{ACKNOWLEDGMENTS}

The authors gratefully acknowledge the assistance of Jennifer Stice, Steve Gum, and Felix Yeh in data collection and analysis. This work was funded in part by NIH grants R01 DC 004086 (DDS) and K02 DC 000136 (DDS). This study was also supported by an NIH training grant (A.L.B.) and an NIDCD research core center grant (P30 DC004665).

\section{REFERENCES}

Apparsundaram S, Ferguson SM, George AlJ, Blakely RD. Molecular cloning of a human, hemicholinium-3-sensitive choline transporter. Biochem. Biophys. Res. Commun. 276:862-867, 2000.

Apparsundaram S, Ferguson SM, Blakely RD. Molecular cloning and characterization of a murine hemicholinium-3-sensitive choline transporter. Biochem. Soc. Trans. 29:711-716, 2001.

Blass S, Meier C, Vohr HW, Schwochau M, Specker C, Burmester GR. The p68 autoantigen characteristic of rheumatoid arthritis is reactive with carbohydrate epitope specific autoantibodies. Ann. Rheum. Dis. 57:220-225, 1998.

BROWN MC. Fiber pathways and branching patterns of biocytinlabeled olivocochlear neurons in the mouse brainstem. J. Comp. Neurol. 337:600-613, 1993.

Bruce LL, Kingsley J, Nichols DH, Fritzsch B. The development of vestibulocochlear efferents and cochlear afferents in mice. Int. J. Dev. Neurosci. 15:671-692, 1997.

Bruce LL, Christensen MA, Warr WB. Postnatal development of efferent synapses in the rat cochlea. J. Comp. Neurol. 423:532$548,2000$.

Comis SD, Guth PS. The release of acetylcholine from the cochlear nucleus upon stimulation of the crossed olivo-cochlear bundle. Neuropharmacology 13:633-641, 1974.

Comis SD, Hayward TL, STOPP PE. Proceedings: a new histological method which facilitates histochemical studies of the cochlea. J. Physiol. 241:63P-65P, 1974.

Emmerling MR, Sobkowicz HM. Acetylcholinesterase-positive innervation in cochleae from two strains of shaker-1 mice. Hear. Res. 47:25-37, 1990.

Ferguson SM, Savchenko V, Apparsundaram S, Zwick M, Wright J, Heilman CJ, Yi H, Levey AI, Blakely RD. Vesicular localization and activity-dependent trafficking of presynaptic choline transporters. J. Neurosci. 23:9697-9709, 2003.

FritzSCH B, NichOLS DH. DiI reveals a prenatal arrival of efferents at the differentiating otocyst of mice. Hear. Res. 65:51-60, 1993.

Galley N, Klinke R, Oertel W, Pause M, Storch WH. The effect of intracochlearly administered acetylcholine-blocking agents on the efferent synapses of the cochlea. Brain Res. 64:55-63, 1973.

Haga T, Noda N. Choline uptake system of rat brain synaptosomes. Biochim. Biophys. Acta. 291:564-575, 1973.

Hoover DB, Ganote CE, Ferguson SM, Blakely RD, Parsons RL. Localization of cholinergic innervation in guinea pig heart by immunohistochemistry for high-affinity choline transporters. Cardiovasc. Res. 62:112-121, 2004.

Hresko RC, Kruse M, Strube M, Mueckler M. Topology of the Glut 1 glucose transporter deduced from glycosylation scanning mutagenesis. J. Biol. Chem. 269:20482-20488, 1994. 
Karnovsky M, Roots L. A 'direct-colouring' thiocholine method for cholinesterases. J. Histochem. Cytochem. 12:219-221, 1964.

Kuhar MJ, Murrin LC. Sodium-dependent, high affinity choline uptake. J. Neurochem. 30:15-21, 1978.

LAEMMLI UK. Cleavage of structural proteins during the assembly of the head of bacteriophage T4. Nature 227:680-685, 1970.

Lips KS, Pfeil U, Haberberger RV, Kummer W. Localisation of the high-affinity choline transporter-1 in the rat skeletal motor unit. Cell Tissue Res. 301:275-280, 2002.

Maison SF, Adams JC, Liberman MC. Olivocochlear innervation in the mouse: immunocytochemical maps, crossed versus uncrossed contributions, and transmitter colocalization. J. Comp. Neurol. 455:406-416, 2003.

Merchan-Perez A, Bartolome MV, Ibanez Ma, Gil-Loyzaga P. Expression of GAP-43 in growing efferent fibers during cochlear development. ORL J. Otorhinolaryngol. Relat. Spec. 55:208-210, 1993.

Merchan-Perez A, Gil-Loyzaga P, Eybalin M, Fernandez Mateos P, BARTOLOME MV. Choline-acetyltransferase-like immunoreactivity in the organ of Corti of the rat during postnatal development. Brain Res. Dev. Brain Res. 82:29-34, 1994.

Misawa H, Nakata K, Matsuura J, Nagao M, Okuda T, Haga T. Distribution of the high-affinity choline transporter in the central nervous system of the rat. Neuroscience 105:87-98, 2001.

NaKaTa K, Okuda T, Misawa H. Ultrastructural localization of highaffinity choline transporter in the rat neuromuscular junction: enrichment on synaptic vesicles. Synapse 53:53-56, 2004.

OKuda T, HaGa T. Functional characterization of the human highaffinity choline transporter. FEBS Lett. 484:92-97, 2000.

Okuda T, Haga T. High-affinity choline transporter. Neurochem. Res. 28:483-488, 2003.

Okuda T, Haga T, Kanai Y, Endou H, Ishihara T, Katsura I. Identification and characterization of the high-affinity choline transporter. Nat. Neurosci. 3:120-125, 2000.

Osen KK, Mugnaini E, Dahl AL, Christiansen AH. Histochemical localization of acetylcholinesterase in the cochlear and superior olivary nuclei. A reappraisal with emphasis on the cochlear granule cell system. Arch. Ital. Biol. 122:169-212, 1984.

Raji-Kubba J, Micevych PE, Simmons DD. Superior olivary complex of the hamster has multiple periods of cholinergic neuron development. J. Chem. Neuroanat. 24:75-93, 2002.
Simmons DD. Development of the inner ear efferent system across vertebrate species. J. Neurobiol. 53:228-250, 2002.

Simmons DD, Raji-Kubba J. Postnatal calcitonin gene-related peptide in the superior olivary complex. J. Chem. Neuroanat. 6:407418, 1993.

Simmons DD, Mansdorf NB, Kim JH. Olivocochlear innervation of inner and outer hair cells during postnatal maturation: evidence for a waiting period. J. Comp. Neurol. 370:551-562, 1996a.

Simmons DD, Moulding HD, ZeE D. Olivocochlear innervation of inner and outer hair cells during postnatal maturation: an immunocytochemical study. Brain Res. Dev. Brain Res. 95:213226, 1996b.

Simmons DD, Raji-Kubba J, Popper P, Micevych PE. Developmentally regulated expression of calcitonin gene-related peptide in the superior olive. J. Comp. Neurol. 377:207-216, 1997.

Simmons DD, Bertolotto C, Kim J, Raji-Kubba J, Mansdorf N. Choline acetyltransferase expression during a putative developmental waiting period. J. Comp. Neurol. 397:281-295, 1998.

Simmons DD, Bertolotto C, Typpo K, Clay A, Wu M. Differential development of cholinergic-like neurons in the superior olive: a light microscopic study. Anat. Embryol. (Berl) 200:585-595, 1999.

Sobkowicz HM, EmmerLing MR. Development of acetylcholinesterase-positive neuronal pathways in the cochlea of the mouse. J. Neurocytol. 18:209-224, 1989.

TUCEK S. Regulation of acetylcholine synthesis in the brain. J. Neurochem. 44:11-24, 1985.

Vetter DE, Mugnaini E. Distribution and dendritic features of three groups of rat olivocochlear neurons. A study with two retrograde cholera toxin tracers. Anat. Embryol. 185:1-16, 1992.

Vetter DE, Adams JC, Mugnaini E. Chemically distinct rat olivocochlear neurons. Synapse 7:21-43, 1991.

WARR WB, GUINAN JJ. Efferent innervation of the organ of Corti: two separate systems. Brain Res. 173:152-155, 1979.

WARR WB, Boche JB, NEely ST. Efferent innervation of the inner hair cell region: origins and terminations of two lateral olivocochlear systems. Hear. Res. 108:89-111, 1997.

YAO W, Godfrey DA. Choline acetyltransferase in cochlear root neurons. Hear. Res. 69:76-82, 1993.

YAO W, GODFREY DA. Immunohistochemical evaluation of cholinergic neurons in the rat superior olivary complex. Microsc. Res. Tech. 41:270-283, 1998. 